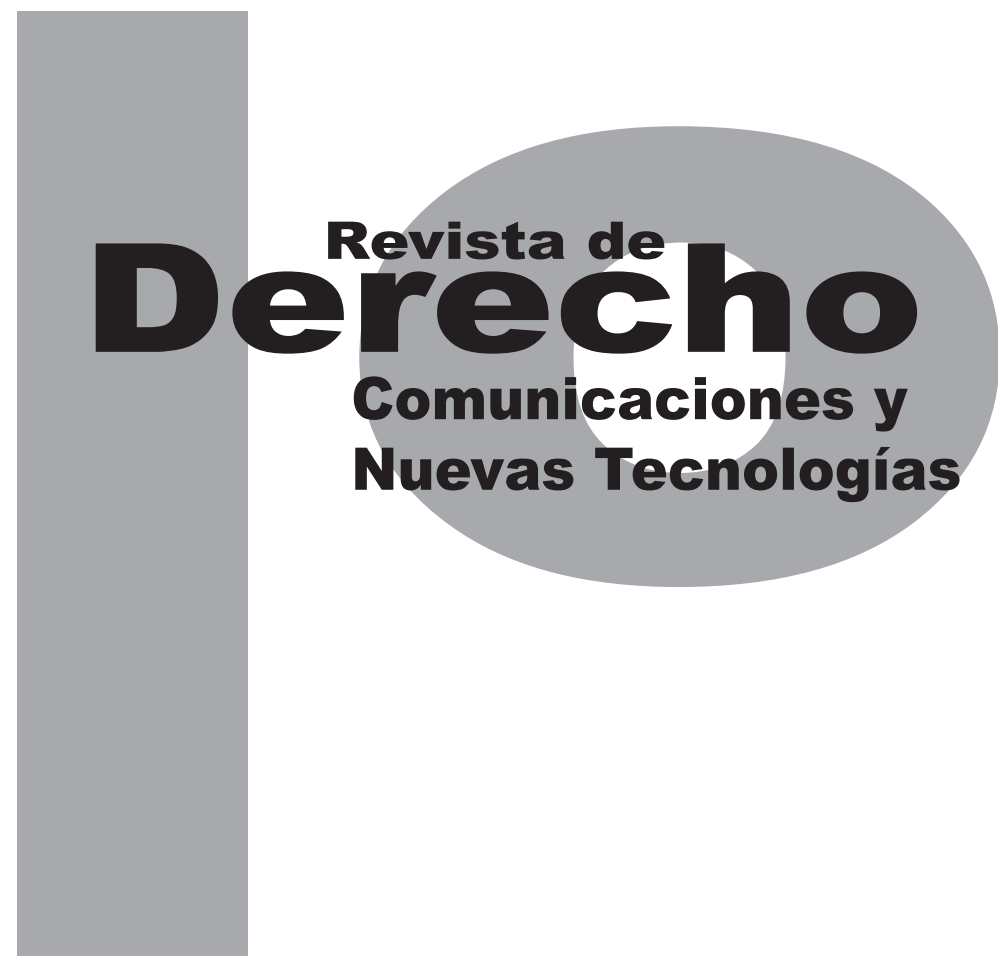

\title{
IMPLEMENTACIÓN DE TECNOLOGÍAS DE LA INFORMACIÓN Y LAS COMUNICACIONES (TIC) EN COLOMBIA
}

\author{
Diana Carolina Valencia Tello
}

Artículo de reflexión

DOI: http://dx.doi.org/10.15425/redecom.14.2015.07

Universidad de los Andes

Facultad de Derecho

Rev. derecho comun. nuevas tecnol.

No. 14, julio - diciembre de 2015. ISSN 1909-7786 


\title{
Implementación de tecnologías de la información y las comunicaciones (TIC) en Colombia
}

\section{Resumen}

Desde el año 2000, el gobierno colombiano identificó la promoción de las tic como un mecanismo fundamental para crear territorios y sociedades más competitivas e incluyentes, buscando la transformación de diversos sectores relevantes para el desarrollo social y económico del país. La implementación de tic en Colombia representa un esfuerzo continuo y permanente de varias administraciones y varios periodos de gobierno interesados en implementar una política pública coherente con las necesidades de la sociedad y del Estado en el siglo xxı. El presente artículo describe la forma como se ha desarrollado la implementación de las tic en Colombia desde el año 2000 hasta el 2014, con el propósito de verificar los principales avances y retos identificados por periodos presidenciales de gobierno.

Palabras clave: gobierno electrónico, políticas públicas, reforma al Estado, tic.

\section{Implementation of information and communications technology (ICT) in Colombia}

\begin{abstract}
Since 2000, the Colombian government has identified the promotion of ICTs as a fundamental mechanism for creating more competitive and inclusive territories and societies, seeking the transformation of various relevant sectors for social and economic development. The implementation of ICT in Colombia represents the continuous and ongoing efforts of various administrations and various periods of government interested in implementing a public policy that is coherent with the needs of society and the state in the twenty-first century. This article describes how the implementation of ICT has developed in Colombia from 2000 to 2014, with the purpose of verifying the main advances and challenges identified by presidential administrations.
\end{abstract}

Keywords: e-government, public policy, state reform, ICT.

\section{Implementação de tecnologias da informação e as comunicações (TIC) na Colômbia}

\section{Resumo}

Desde o ano 2000, o governo colombiano identificou a promoção das TIC como um mecanismo fundamental para criar territórios e sociedades mais competitivas e de inclusão, buscando a transformação de diversos setores relevantes para o desenvolvimento social e econômico do país. A implementação das TIC na Colômbia representa um esforço contínuo e permanente de várias administrações e vários períodos de governo interessados em implementar uma política pública coerente com as necessidades da sociedade e do Estado no século XXI. O presente artigo descreve a forma como tem se desenvolvido a implementação das TIC na Colômbia desde o ano 2000 até 2014, com o propósito de verificar os principais avanços e desafios identificados por períodos presidenciais de governo.

Palavras-chave: governo eletrônico, políticas públicas, reforma ao Estado, TIC 


\title{
Implementación de tecnologías de la información y las comunicaciones (TIC) en Colombia*
}

\author{
Diana Carolina Valencia Tello**
}

\section{SUMARIO}

Introducción - I. GENERALIDADES SOBRE EL GOBIERNO ELECTRÓNICO - II. IMPLEMENTACIÓN DEL GOBIERNO ELECTRÓNICO EN COLOMBIA - A. Andrés Pastrana Arango (1998-2002) - B. Álvaro Uribe Vélez (2002-2010) - C. Juan Manuel Santos (2010-2014) - III. CONLCUSIONES - Referencias.

Cómo citar este artículo: Valencia Tello, D. C. (Diciembre, 2015). Implementación de tecnologías de la información y las comunicaciones (TIC) en Colombia. Revista de Derecho, Comunicaciones y Nuevas Tecnologías, 14. Universidad de los Andes (Colombia).

Este artículo hace parte de la línea de investigación sobre Derecho de Estado, del Núcleo de Constitucionalismo y Democracia de la Facultad de Derecho de la Universidad Federal del Paraná. Fue financiado por la CAPES.

** Profesora visitante de la Facultad de Derecho de la Universidad Federal del Paraná. Abogada, con especialización en Derecho Administrativo y Ambiental de la Universidad del Rosario. Maestría, doctorado y postdoctorado en Derecho de Estado en la Universidad Federal del Paraná (Brasil). Correo: dianacvt@hotmail.com. 
Introducción

En el siglo xxı las tecnologías de la información y las comunicaciones (TIC) tienen un papel fundamental en el desarrollo económico y social de los países y las comunidades. Las tic potencializan la comunicación y la cooperación entre diversos actores, ayudando a la creación de redes, alrededor de objetivos comunes previamente identificados. Las redes, como nueva forma de organización social, están transformando las relaciones de poder entre el Estado y las sociedades de forma permanente y continua.

En los últimos años, las sociedades alrededor del mundo están reclamando cada vez más y mejores servicios públicos por parte del Estado. También están reclamando mayor capacidad de gestión, efectividad e innovación, pues las viejas estructuras burocráticas, jerárquicas, autoritarias y cerradas a las necesidades de la sociedad y de los ciudadanos no consiguen dar respuestas efectivas a las diferentes problemáticas existentes en la actualidad.

Las TIC representan nuevos desafíos para los gobiernos, quienes de ahora en adelante deben mejorar los canales de comunicación con la ciudadanía mediante la utilización y aprovechamiento de las ventajas de estas para optimizar la gestión gubernamental en todos los niveles; el no hacerlo coloca en riesgo la relevancia del propio Estado en las diferentes redes de poder que atraviesan las sociedades y los territorios.

La gran cantidad de información y de servicios que genera el Estado es una de las principales dificultades para mejorar la coordinación y comunicación entre entidades, ya que las tradicionales estructuras administrativas jerárquicas y autoritarias no permiten fácilmente el flujo de información. Así, para implementar las tıc, el Estado debe primero reformular las viejas estructuras de poder, propiciando estructuras más horizontales e interconectadas que faciliten la comunicación en la administración pública y de esta con la ciudadanía.

En este contexto, las TIC, aplicadas a la mejora de la gestión pública, pueden potencializar las capacidades del gobierno para descentralizar de forma responsable el cumplimiento de sus diferentes funciones y actividades, manteniendo un control centralizado y ayudando a mejorar la eficiencia, la coordinación y la coherencia en las diferentes actividades que debe cumplir el Estado.

Aquí es importante resaltar que el gobierno colombiano desde el año 2000 ha identificado la promoción de las tic como un mecanismo fundamental para crear territorios y sociedades más competitivas e incluyentes, buscando que a través de la tecnología se logren transformaciones contundentes y positivas en la gestión de diversos sectores relevantes para el desarrollo social y económico del país.

La implementación de las tic en Colombia representa un esfuerzo continuo y permanente de varias administraciones y periodos de gobierno, interesados en una política pública coherente con las necesidades de la sociedad y del Estado en el siglo xxı. Esta política pública se caracterizó 
desde un inicio por tener una fuerte estructura conceptual y metodológica que ha permitido mayor coordinación y comunicación entre diversos actores de diferentes niveles de gobierno para alcanzar las metas propuestas.

Teniendo en cuenta lo anterior, el presente artículo busca describir la forma como se ha desarrollado la implementación de las tic en Colombia desde el año 2000, con el fin de verificar los principales avances y retos identificados por periodos de gobierno hasta el año 2014.

Para realizar este estudio, primero se analizarán algunas generalidades sobre el gobierno electrónico, para después estudiar la forma como se ha implementado la política pública de gobierno electrónico en Colombia desde el año 2000 hasta el 2014. La metodología adoptada es de naturaleza cualitativa y descriptiva, mediante el análisis del ordenamiento jurídico vigente, documentos oficiales, libros, periódicos, artículos en revistas indexadas y portales electrónicos reconocidos.

\section{GENERALIDADES SOBRE EL GOBIERNO ELECTRÓNICO}

El Estado, en el siglo xxI, necesita realizar transformaciones al interior de las entidades para que las estructuras sean más horizontales y eficientes, mediante la organización de procesos que puedan comunicarse permanentemente vía interconexiones internas que permitan mejorar los flujos de información necesarios para la coordinación y la cooperación institucional.
Para que esto sea posible, las entidades públicas también necesitan fortalecer la capacidad gerencial de los equipos de trabajo, lo que implica obligatoriamente una redistribución del poder y de los recursos públicos dentro de las estructuras del Estado. Para que el Estado sea más eficiente es necesario descentralizar, desconcentrar y delegar competencias y funciones, pero sin perder el control y la vigilancia que desde el centro debe dar coherencia a las diferentes actividades.

En este contexto, las TIC, aplicadas a la mejora de la gestión pública, pueden potencializar las capacidades del gobierno para descentralizar de forma responsable el cumplimiento de las diferentes funciones y actividades, manteniendo el control centralizado y ayudando a mejorar la eficiencia, la coordinación y la coherencia en las diferentes actividades que debe cumplir el Estado (Valencia, 2015).

Según definición del Banco Mundial (2013), el gobierno electrónico es el uso que las entidades públicas hacen de las Tıc, tales como redes, Internet y tecnología móvil, las cuales tienen la habilidad de transformar las relaciones con ciudadanos, empresas y otras agencias de gobierno. Estas tecnologías pueden ser usadas para diversos fines, entre los cuales se encuentran: entregar mejores servicios a los ciudadanos, mejorar las relaciones con las empresas y las industrias, fortalecer las capacidades de los ciudadanos mediante el acceso a la información o hacer más eficiente la gestión del gobierno. Las ventajas de estas acciones pueden reflejarse en la disminución de la corrupción, en el aumento 
de la transparencia y de los ingresos, en la disminución de los costos de los diferentes procesos administrativos, entre otros.

Tradicionalmente, la interacción entre los ciudadanos, las empresas y las entidades gubernamentales tenía lugar en las oficinas del gobierno. En la actualidad, con las TIC es posible establecer centros de servicio más cercanos a los usuarios. Estos centros pueden estar localizados en sitios estratégicos o estar disponibles mediante el uso de computadores o dispositivos móviles de uso personal en las casas o en las oficinas. Así, el gobierno electrónico busca mejorar de diferentes formas las relaciones entre el gobierno y los ciudadanos (G2C), el gobierno y las empresas (G2B) y las interacciones entre las diferentes entidades o agencias de gobierno (G2G).

En la actualidad, es imperativo que los Estados tengan presencia en Internet, y suministren por lo menos información y servicios básicos a los ciudadanos, buscando construir confianza en el gobierno, mostrar una gestión transparente y disposición para mejorar la comunicación con la ciudadanía y los grupos de interés. Por lo tanto, los gobiernos deben facilitar el acceso a las nuevas tecnologías, en vez de limitarlas.

En este contexto, el gobierno electrónico es una herramienta básica para construir una estrategia sustentable de desarrollo que permita la integración de diferentes servicios y entidades públicas en tiempo real; maximizar la cooperación, la eficiencia, la eficacia y la distribución de los recursos; y una mayor interacción de los usuarios de los servicios públicos con el Estado, en tiempo real. El gobierno electrónico puede permitir, en el largo plazo, integrar las diferentes metas económicas, sociales y medio ambientales de los gobiernos de forma coherente y coordinada, en determinados periodos de tiempo. Al respecto, el informe de 2012 de las Naciones Unidas (onU) sobre el gobierno electrónico afirma lo siguiente:

El gobierno electrónico tiene una función muy importante en la actualidad, y la seguirá teniendo en el futuro. (...) En ese contexto, los gobiernos nacionales deben comprender que las vías económicas, sociales y ambientales deben adaptarse para desarrollar o reformar sus marcos estratégicos, con el fin de lograr resultados que fomenten el desarrollo sostenible. El enfoque básico estratégico debe dar por resultado, principalmente, la aceptación de la importancia de las interrelaciones entre los aspectos económicos, sociales y ambientales del desarrollo. Una vez más, la función del gobierno se redefine para reformar los sistemas de gobernanza mediante los cuales se ofrecen los servicios de una manera tal que se maximiza el desarrollo y se minimiza la degradación de los recursos naturales. Una aproximación holística de la gobernanza debe considerar los aspectos de distribución y eficacia de las políticas sectoriales y sus resultados, las agendas nacionales de desarrollo y los acuerdos de cooperación internacional, de manera que las soluciones derivadas sean sostenibles en el futuro. (Pág. 2)

Sin embargo, para implementar políticas efectivas de gobierno electrónico los Estados deben desarrollar diversas capacidades, en diferentes niveles de gobierno, lo cual requiere tiempo y 
voluntad política, así como estrategias en el corto, mediano y largo plazo que ayuden a transformar la gestión pública, conforme las nuevas necesidades del siglo xxI.

Adicionalmente, para que el gobierno electrónico sea efectivo es necesario reducir las barreras digitales existentes entre los diversos grupos de la sociedad, mediante la generación de políticas de inclusión que promuevan el desarrollo de las capacidades necesarias en los ciudadanos para el uso de las TIc, además de políticas de participación ciudadana mediante dichas tecnologías.

En mi concepto, la política pública sobre gobierno electrónico desarrollada en Colombia desde el año 2000 es un buen ejemplo de implementación de estrategias de corto, mediano y largo plazo orientadas a transformar la gestión pública de cara al siglo xxı. También es un caso interesante de estudio para verificar las oportunidades y dificultades que los gobiernos y las administraciones públicas enfrentan al momento de implementar efectivamente las TIc.

\section{IMPLEMENTACIÓN DEL GOBIERNO ELECTRÓNICO EN COLOMBIA}

El gobierno colombiano desde el año 2000 identificó la promoción de las tıc como un mecanismo fundamental para crear territorios y sociedades más competitivas e incluyentes, buscando que a través de la tecnología se logren transformaciones contundentes y positivas en la gestión de diversos sectores relevantes para el desarrollo social y económico del país.
No obstante lo anterior, es claro que las tic no logran transformaciones milagrosas por sí solas, sino que deben darse cambios en el conjunto de la sociedad y las instituciones para que las nuevas tecnologías puedan ser efectivamente aplicadas y utilizadas en todo su potencial.

La política pública de implementación de las TIC en Colombia ha estado acompañada de varias transformaciones estructurales en la administración pública y en la sociedad, tendientes a la creación de competencias y habilidades en funcionarios públicos y ciudadanos para el buen uso de tales tecnologías en el territorio nacional. De esta forma se puede afirmar que Colombia ha buscado mejorar y fortalecer la gestión de las entidades públicas mediante la implementación de políticas públicas cuidadosamente diseñadas, que reestructuran procesos y procedimientos administrativos utilizando las TIC, lo que está ayudando a construir un nuevo espacio más participativo e incluyente en las diversas entidades del Estado.

En 2012, dicha política fue premiada en el Mobile World Congress de Barcelona, evento en donde se entregó al Ministerio de Tecnologías de la Información y las Comunicaciones (en adelante MinTIC) el galardón Government Leadership Award 2012 (MinTIC, 2014).

Los logros en materia de implementación de las TIC en Colombia no son pocos. Según el MinTIC (2014), Colombia será el primer país latinoamericano en contar con servicio de Internet de alta velocidad en el $100 \%$ de su territorio. Para lograr este objetivo, desde el 2010 el MinTIC 
desarrolló el Plan Vive Digital, el cual permitió que el país pasara de 2.2 millones de conexiones a Internet a 8.8 millones de conexiones en tan solo cuatro años, gracias, en parte, a que el Plan se propuso conectar a todos los municipios a dicho servicio. Así, en 2010 el Gobierno nacional registraba 200 municipios conectados y en 2014 se reportaron más de 1.078 municipios conectados a Internet.

Los logros del MinTIC no se limitan al fortalecimiento de la infraestructura en tIc; también se han registrado importantes mejoras en las áreas de prestación de servicios, nuevas aplicaciones, soporte a usuarios y promoción de ecosistemas digitales. Esto porque como ya fue mencionado, la política en esta materia busca la transformación de la sociedad y las instituciones, mediante la creación y la mejora de competencias y habilidades en funcionarios públicos y ciudadanos para el buen uso de las tic en el territorio nacional.

Para estudiar la forma como se ha construido la política pública de gobierno electrónico en Colombia desde el año 2000 al 2014, se analizarán los avances y las dificultades presentadas en los tres últimos periodos de gobierno, teniendo en cuenta que en el país el periodo de gobierno presidencial es de cuatro años y que en el lapso de tiempo en estudio fue permitida una única reelección presidencial.

Así, a continuación se analizarán los periodos presidenciales de Andrés Pastrana Arango (1998-2002), Álvaro Uribe Vélez (2002- 2010) y Juan Manuel Santos Calderón (2010-2014) con sus principales retos y logros.

\section{A. Andrés Pastrana Arango (1998-2002)}

A finales de la década de los años noventa el país no tenía importantes tecnologías implementadas; el inicio del gobierno electrónico comienza en el mandato del presidente Pastrana, porque fue durante este que se estableció el uso de las tic como uno de los pilares de la agenda presidencial.

En el año 2000 se inició formalmente el proceso de consolidación de una política pública para la implementación de Tıc, caracterizada por el establecimiento de reglas, directrices y arreglos institucionales como parte de una estrategia de masificación del uso de las Tic, coherente y continua dentro del Estado. Lo anterior implicó reformas institucionales de fondo que ayudaran a acoplar la estructura estatal a la nueva revolución tecnológica que estaba en formación.

El documento Conpes ${ }^{1} 3072$ de 2000 creó el programa Agenda de Conectividad, responsable de masificar el uso de las tecnologías de la información en Colombia, buscando aumentar la competitividad del sector productivo, modernizar las instituciones públicas y de gobierno, y socializar el acceso a la información.

\footnotetext{
1 Consejo Nacional de Política Económica y Social. Es la máxima autoridad nacional de planeación y se desempeña como organismo asesor del Gobierno en todos los aspectos relacionados con el desarrollo económico y social del país. Para lograrlo, coordina y orienta a los organismos encargados de la dirección económica y social, a través del estudio y aprobación de documentos sobre el desarrollo de políticas generales que son presentados en sesión. El Departamento Nacional de Planeación desempeña las funciones de Secretaría Ejecutiva del Conpes y Conpes Social, y por lo tanto es la entidad encargada de coordinar y presentar todos los documentos para discutir en sesión.
} 
Este documento Conpes ayudó a articular diferentes iniciativas, las cuales tenían en principio cuatro objetivos principales. Primero, desarrollar una nueva economía (E-conomía²); segundo, la construcción de un Estado más moderno y eficiente; tercero, la necesidad de la universalización del acceso a la información; y, finalmente, la importancia de adquirir y utilizar eficazmente el conocimiento. Todos estos elementos se entendían como fundamentales para el desarrollo de la sociedad del siglo xxI.

Con base en las directrices establecidas en el Conpes 3072 se procuró aumentar la competitividad del sector productivo, modernizar las instituciones públicas y socializar el acceso a la información, mediante el establecimiento de objetivos y estrategias orientadas a impulsar el desarrollo a través del uso de las tıc en tres sectores principales: la comunidad, el sector productivo y el Estado. De esta forma, desde el año 2000 se institucionalizó una política pública con un marco conceptual fuerte que ayudó a direccionar las diversas estrategias y actividades que debían ser realizadas para la implementación efectiva e integral de las TIc en Colombia.

Durante esta primera etapa, el programa Agenda de Conectividad dependía directamente del Presidente de la República (Decreto 127 de 2001). En consecuencia, desde allí se establecieron varias directrices con el objetivo de implementar las tıc en las entidades públicas. La Directiva Presidencial 002 de 2000 es una de ellas. La

2 La E-conomía es definida como aquella que está basada en la interacción de las tecnologías de la información (TI) y la economía tradicional. importancia dada por el presidente Pastrana al programa Agenda de Conectividad fue un poderoso mensaje para entidades y funcionarios del gobierno nacional, al evidenciar que existía voluntad política para realizar reformas dentro de la administración pública, buscando mayor gobernabilidad y mayor comunicación con la ciudadanía.

Aun así, el programa Agenda de Conectividad era nuevo, contaba con poca infraestructura y poco soporte teórico; además, en este momento no existían buenos mecanismos que ayudarán a controlar o monitorear el cumplimiento de las directivas presidenciales por parte de las entidades públicas. Teniendo en cuenta las dificultades encontradas para la efectiva implementación del gobierno electrónico, el consenso sobre la necesidad de realizar reformas dentro de la administración pública para mejorar la coordinación entre funcionarios y entidades, así como también para mejorar la estrategia de información no se hizo esperar.

\section{B. Álvaro Uribe Vélez (2002-2010)}

Durante las dos administraciones presidenciales de Álvaro Uribe (2002-2010), la implementación de las tic nuevamente fue uno de los pilares de la agenda de gobierno, como puede apreciarse en el Plan Nacional de Desarrollo 20032006 (Ley 812 de 2003) y el Plan Nacional de Desarrollo 2006-2010 (Ley 1151 de 2007). Adicionalmente, al inicio de su administración el Congreso aprobó la Ley para la Renovación de la Administración Pública (Ley 790 de 2002), la cual estableció la obligación del gobierno nacio- 
nal de promocionar el gobierno electrónico en todo el país.

El documento Conpes 3248 de 2003 estableció el Programa de Renovación de la Administración Pública (PRAP) que tuvo a cargo apoyar y coordinar la realización de profundos cambios al interior de esta y las entidades, con el fin de promover la construcción de una administración pública centrada en los ciudadanos, en cuya concepción, ejecución y control participa activamente la comunidad. La implementación de las Tic como soporte del gobierno electrónico fue desde el inicio una de las herramientas identificadas como esenciales para acercar el Estado al ciudadano.

Debido a que la administración pública necesitaba de profundas transformaciones institucionales y culturales para modificar estructuras burocráticas jerárquicas y autorreferidas, que poco se comunicaban con la ciudadanía, antes de oficializar el programa de gobierno electrónico el Estado colombiano ya estaba trabajando conjuntamente varias reformas internas que comprendían cuatro componentes principales: reestructuración de la administración pública, reforma en la gestión del empleo público, fortalecimiento de los sistemas transversales de gestión e integración de la información para la gestión pública.

Una de las reformas fundamentales para mejorar los cuatro componentes previamente enunciados fue la implementación de los sistemas de gestión y control en las entidades públicas. Las normas sobre control interno y gestión de la ca- lidad ayudaron a estructurar la administración pública con base en procesos direccionados a la entrega de productos y servicios de calidad a los ciudadanos, y a mejorar la comunicación entre diversos actores relevantes (públicos y privados), previamente identificados, para el buen desarrollo de los procesos internos. La mejora en la comunicación fue posible mediante la implementación de procesos y mecanismos de atención a la ciudadanía y seguimiento de su nivel de satisfacción con relación a los productos y servicios entregados.

Como vimos en el periodo presidencial de Andrés Pastrana, una de las limitaciones encontradas al momento de implementar la política de tic en las entidades públicas fue la falta de mecanismos para gestionar y controlar el cumplimiento de las directivas presidenciales en esta materia. Esto porque en administraciones estructuradas bajo un modelo jerárquico y autoritario la comunicación entre dependencias y funcionarios públicos es limitada, y los procesos no cuentan con parámetros bien definidos que permitan una efectiva articulación entre los diferentes actores. Tampoco disponen de mecanismos de seguimiento y control sobre el cumplimiento de las directrices establecidas, lo que impide la ejecución en la práctica de cualquier programa de gobierno.

Así, la implementación obligatoria de sistemas de gestión y control en las entidades públicas, conforme a parámetros bien definidos en las leyes y en los procesos internos de las entidades, ayudó a conformar equipos de trabajo que traspasan las dependencias, a documentar y 
gestionar los flujos de información dentro de los procesos para mejorar no solo la gestión y los resultados de las entidades, sino también la rendición de cuentas a la ciudadanía mediante la entrega de información clara y oportuna sobre el funcionamiento y los avances de la entidad.

La puesta en marcha de los sistemas de gestión y control fue posible mediante la creación de estándares de obligatorio cumplimiento para todas las entidades públicas del nivel nacional, departamental y municipal, los cuales fueron adoptados oficialmente mediante los decretos 4110 de 2004 y 1599 de 2005. Entre ellos, la obligación de implementar dichos sistemas en un plazo no superior a los 24 meses, responsabilidad que quedó en cabeza de la autoridad máxima de la entidad, quien puede delegarla en otros funcionarios.

Los sistemas de gestión y control permitieron a las entidades públicas en todos los niveles organizar la administración pública por procesos, procedimientos e indicadores de gestión, entre otros componentes, lo que ayudó a mejorar la comunicación interna y a organizar la información necesaria para facilitar el diálogo entre funcionarios públicos y ciudadanos. El cumplimiento de las normas sobre los sistemas de gestión y control es evaluado permanentemente por la Contraloría General de la República, que presenta informes anuales sobre control interno a la sociedad y al Congreso de la República, conforme lo dispone la Constitución.

La experiencia colombiana evidencia que para avanzar en el gobierno electrónico es necesario reestructurar internamente la administración pública, buscando mejores prácticas administrativas, estructuras más horizontales e interconectadas, con información bien organizada, enfocada a la atención al ciudadano y con funcionarios públicos altamente competentes.

Sin embargo, también es verdad que modificar viejas prácticas y tradiciones toma tiempo, requiere de voluntad política y trabajo constante, que debe ser supervisado de forma permanente, sistemática y eficaz. Los resultados, por tanto, son diversos, especialmente si se tiene en cuenta que en algunas entidades los sistemas de gestión y control se adoptan "formalmente", es decir, sin ir acompañados de cambios en la cultura organizacional o en la atención al ciudadano. Esta formalidad impide que los procesos internos generen transformaciones importantes en la administración pública o en la sociedad.

Durante el mandato de Uribe el programa Agenda de Conectividad, encargado de direccionar la masificación del uso de las tic, se trasladó al Ministerio de Comunicaciones, en donde se desarrolló y estructuró la estrategia Gobierno en Línea, la cual fue formulada sobre un fuerte marco conceptual y metodológico, con base en seis principios: 1. Gobierno centrado en el ciudadano, 2. Visión unificada del Estado, 3. Acceso equitativo y multicanal, 4. Gobierno en línea es más que tecnología, 5. Protección de la información del individuo y 6. Credibilidad y confianza del gobierno en línea. Como ejes de acción se establecieron tres componentes principales: 1. Mejorar la provisión de servicios a los ciudadanos y a las empresas, 2. Fortalecer la 
transparencia del Estado y la participación ciudadana, y 3. Mejorar la eficiencia del Estado.

La estrategia Gobierno en Línea incluye el desarrollo de metodologías que determinan claramente la información mínima que deben contener las páginas web de las entidades, así como también los servicios que se deben ofrecer utilizando las TIC. La metodología creada durante este periodo establece un proceso gradual y evolutivo de implementación que cuenta con cinco fases de desarrollo: información, interacción, transacción, transformación y democracia electrónica. Estas fases se basan en una visión unificada del Estado, que busca interconectar por procesos a las entidades con la finalidad de evitar trámites y demoras innecesarias a los ciudadanos.

Adicionalmente, se creó la Comisión Intersectorial de Políticas de Gestión de Información de la Administración Pública (coinfo), como instancia de coordinación encargada de la definición de estrategias y objetivos en el campo de los sistemas de información del sector público. Su propósito fue la optimización de la calidad, la accesibilidad y el uso adecuado de la información producida por las instituciones del Estado colombiano. En este periodo de gobierno dicha Comisión fue presidida por el Vicepresidente de la República, y estaba compuesta por funcionarios públicos de alto rango de todos los sectores de la administración, con la finalidad de articular, coordinar, integrar y definir mecanismos de optimización en la producción y control de la información, lo que incluye también la implementación de estrategias de gobierno electrónico.
Para garantizar la implementación efectiva de Gobierno en Línea se determinaron plazos perentorios de cumplimiento para las entidades en cada una de las fases de la estrategia, mediante la promulgación del Decreto 1151 de 2008. Adicionalmente, se fijó en los ministerios (en el orden nacional) y en las gobernaciones (en el orden territorial), la responsabilidad de coordinar la implementación del Gobierno en Línea en las entidades pertenecientes a su sector, buscando mayor interconexión y comunicación entre las entidades que participan en un mismo proceso.

Así, cada sector tuvo funcionarios a cargo de Gobierno en Línea para verificar en las respectivas páginas web el cumplimiento de los requisitos y servicios mínimos, generando calificaciones que fueron retroalimentadas a los jefes de cada entidad, y que permitieron verificar por periodos el grado de avance de las entidades en la efectiva implementación del gobierno electrónico, con datos confiables e información oportuna. Este oportuno seguimiento dejó en evidencia que el Decreto 1151 de 2008 estableció plazos demasiado cortos para los propósitos señalados.

La evaluación realizada por la Universidad de los Andes (2009), mostró que las entidades del orden nacional lograron alcanzar el 55\% de las metas establecidas y las entidades locales solo el 10\%. Según el MinTIC (2011a) esta evaluación evidenció la necesidad de aterrizar las metas de Gobierno en Línea a la realidad y los recursos de las entidades públicas, especialmente en el orden territorial. 
No obstante, se debe resaltar que durante el gobierno de Uribe se realizaron grandes avances en materia de gobierno electrónico. Entre los más significativos se encuentran: el Portal del Estado Colombiano (PEC), la creación del Portal Único de Contratación (PUc), la consolidación de la estrategia Gobierno en Línea, el Sistema Único de Información y Trámites (suit), la Intranet Gubernamental, entre otros.

Según el MinTIC (2011a), el PEC, como centro de información de trámites y servicios para los colombianos, centraliza la información de las entidades públicas nacionales y locales con el fin de facilitar a la ciudadanía el acceso a la información; entre el 2006 y el 2010 reporta 1.798 trámites del orden nacional y 1.487 trámites del orden territorial publicados; y el número de visitas al portal pasó de un promedio mensual de 119.195 en el 2006 a 573.099 en el 2010.

Es importante resaltar también el número de capacitaciones realizadas a funcionarios públicos y contratistas en materia de Gobierno en Línea, con el fin de sensibilizar sobre la importancia de las TIc en la gestión pública. Según el MinTIC (2011a), entre el 2007 y el 2010 se capacitaron 20.761 servidores públicos y contratistas del orden nacional y 77.091 servidores públicos y contratistas del orden territorial.

Los avances en materia de gobierno electrónico fueron ampliamente reconocidos por la comunidad internacional en su momento. En el informe de 2010 sobre gobierno electrónico de la onu, Colombia ocupó el primer lugar entre los países de América Latina y el Caribe, lo que le permitió ubicarse en la posición 31 dentro de la clasificación mundial. Según la anterior clasificación realizada en 2008, el país en dos años mejoró 21 posiciones, lo que demuestra el esfuerzo realizado por el Estado colombiano para la efectiva implementación del gobierno electrónico en la administración pública.

\section{Juan Manuel Santos (2010-2014)}

Durante el primer periodo presidencial de Juan Manuel Santos también se adoptó la política de implementación de tıc como uno de los pilares de su agenda, según consta en el Plan Nacional de Desarrollo 2010-2014 (Ley 1450 de 2011). Teniendo en cuenta la importancia de las TIC para la promoción del desarrollo social y económico del país, el presidente Santos cambió la denominación del Ministerio de Comunicaciones por la de Ministerio de Tecnologías de la Información y las Comunicaciones (MinTIC), lo que significó un fortalecimiento institucional importante para la implementación y desarrollo de las TIc en Colombia.

Al inicio de este periodo de gobierno era evidente que Colombia había avanzado en la prestación de servicios e información en línea, gracias al trabajo realizado en el marco del programa Agenda de Conectividad, pero la penetración de Internet en el país todavía era baja, razón por la cual era importante desarrollar una estrategia para mejorar la infraestructura tecnológica del país de cara al siglo xxı.

En este contexto, en 2010 se creó el Plan Vive Digital, con el objetivo principal de crear empleo 
y generar riqueza mediante la promoción de la revolución digital, que se propuso llevar Internet a los 39 millones de colombianos de los estratos más bajos (1, 2 y 3), es decir, al $86 \%$ de la población, y a las pequeñas y medianas empresas que representan el $96 \%$ de las empresas colombianas según datos de las Cámaras de Comercio. Para esto trabajaría de forma paralela en cuatro frentes principales: infraestructura, servicios, aplicaciones y usuarios. Este ambicioso Plan cuenta con mecanismos de medición para evaluar por periodos de tiempo el grado de avance de las metas establecidas.

También se creó el Consejo Nacional Digital, liderado por el Presidente de la República, y compuesto por funcionarios públicos de alto rango de todos los sectores así como por representantes de la sociedad en calidad de invitados; su objetivo: seguir paso a paso las iniciativas del Plan Vive Digital. Aquí se señalan los principales logros alcanzados por el MinTic en los primeros cuatro años, conforme el Informe de Gestión presentado al Congreso de la República en el 2014, destacando los principales avances en infraestructura, servicios, aplicaciones y usuarios.

Para comenzar, entre el 2010 y el 2014 Colombia pasó de tener 2.2 millones de conexiones de Internet a más de 8.8 millones; esto lo convirtió en el primer país de Latinoamérica con Internet de alta velocidad en todos los municipios, ya que en 2014, un total de 1.078 municipios (el $96 \%$ ) estaban conectados por fibra óptica. Este proyecto tuvo una inversión total de $\$ 415.837$ millones de pesos, fueron instalados $14.000 \mathrm{ki}-$ lómetros de fibra óptica a lo largo del territorio nacional y en la actualidad 1.226 instituciones públicas cuentan con el servicio de Internet de banda ancha gratuito durante cinco años.

Entre los retos principales de este proyecto se encuentra conectar al resto de municipios con mayor dificultad de acceso y con problemas de orden público. También el MinTIC resalta que es importante realizar un seguimiento estricto a la infraestructura durante la fase de operación, para garantizar la calidad de la red de fibra óptica y el servicio de acceso a Internet de acuerdo con los indicadores pactados en el contrato.

También es importante resaltar aquí la mejora realizada en las conexiones internacionales mediante la ampliación de infraestructura de cables submarinos, gracias al potencial de crecimiento del tráfico de Internet en Colombia que visibilizó el Plan Vive Digital y que motivó al sector privado a invertir en cuatro nuevos cables submarinos que mejoran de forma importante la conexión del país con el mundo. En agosto de 2010 Colombia contaba con cinco cables submarinos que llegaban por el mar Caribe, en marzo de 2013 se anunciaron tres más que fueron instalados también por el mar Caribe, y en junio de 2015 se dio la noticia de la entrada en operación del cable submarino del Pacífico, para un total de nueve que ayudarán a mejorar la conexión de Colombia con el mundo por más de veinte veces, según cálculos del MinTIC.

Finalmente, en materia de infraestructura debo resaltar la intranet gubernamental, que es la plataforma que conecta a las diversas entidades públicas y les permite compartir e intercam- 
biar información, realizar actividades y procesos conjuntos, desarrollar trámites y servicios en línea y facilitar el acceso de todos los ciudadanos a información pública. Esta plataforma está compuesta por los siguientes componentes: red de alta velocidad, centro de datos, centro de contacto con el ciudadano y la plataforma de interoperatividad. En agosto de 2010 había 116 entidades vinculadas a la intranet y 40 entidades del orden nacional publicaban servicios de intercambio de información; en marzo de 2014, las entidades vinculadas a la intranet sumaban 142 y 64 entidades del orden nacional publicaban servicios de intercambio de información. Estos datos evidencian el gran avance logrado en los últimos años para mejorar los flujos de información y la cooperación entre las entidades públicas en Colombia.

En materia de servicios, teniendo en cuenta el fortalecimiento de la infraestructura TIC en el país, el MinTIC se propuso masificar las terminales, es decir, el número de computadores, tabletas, teléfonos inteligentes, etc., lo que implicaba duplicar el número de terminales en cuatro años. Como resultado, las 16,8 terminales por cada 100 habitantes que se registraron en el 2010 pasaron en marzo de 2014 a 34 terminales por cada 100 habitantes. Esto fue posible gracias a la reducción de aranceles sobre las terminales, lo que permitió que Colombia cuente con las terminales más baratas del continente.

EI MinTIC también ha realizado desde el 2010 la mayor entrega gratuita de computadores y tabletas a las entidades educativas en todo el país: 669.000 computadores y 1.132 .000 ta- bletas apoyan procesos de formación e información en 34.191 sedes educativas, bibliotecas y casas de cultura pública, que a su vez permiten que 7.391.612 estudiantes cuenten con herramientas tecnológicas para su estudio. El monto de la inversión fue de \$611.048 millones de pesos. Según datos del Ministerio de Educación, Colombia en 2014 tiene una relación promedio de 11 estudiantes por PC, lo que posibilita que cada vez más asignaturas puedan usar las TIC para mejorar la calidad de la educación.

La estrategia de entrega gratuita de computadores estuvo acompañada de un programa de formación de maestros para la masificación de las TIC y la mejora de la calidad educativa. Entre agosto de 2010 y marzo de 2014 el MinTIC formó durante más de 150 horas presenciales a 38.372 docentes ubicados en 13.787 sedes educativas. Este proyecto tuvo un costo total de $\$ 70.235$ millones de pesos.

Con relación a la inclusión digital, el Informe de gestión de 2014 del MinTIC resalta que en 2010 solo el $27 \%$ de los hogares colombianos y el $7 \%$ de las pequeñas y medianas empresas estaban conectados a Internet, y a través del Plan Vive Digital se propuso conectar al 50\% de los hogares colombianos y al $50 \%$ de las pequeñas y medianas empresas, al 2014. Esta meta fue cumplida ampliamente, ya que en cuatro años del programa se logró conectar al 60,6\% de las pequeñas y medianas empresas, lo que representa un incremento de $757 \%$ respecto del 2010. También se alcanzó un $90 \%$ de crecimiento en la conexión de los hogares y se llevó Internet a las zonas rurales al establecer 7.621 Kioskos di- 
gitales en centros rurales con población de más de 100 habitantes.

En las ciudades, el Plan Vive Digital ayudó a crear subsidios de Internet de banda ancha para más de dos millones de familias de los estratos 1 y 2, y habilitó Puntos Vive Digital que realizan capacitaciones estratégicas para la ciudadanía sobre el uso de Internet.

En materia de aplicaciones, desde el 2014 Colombia cuenta con 17 Vivelabs o laboratorios de creación de contenidos digitales, donde se apoya el talento y la creatividad de jóvenes desarrolladores de animación, videos, plataformas y videojuegos, entre otras. Esto, sumado a las iniciativas apps.co, ha dado vida a la mayor red de emprendedores de Latinoamérica, donde 55.000 colombianos con proyectos y modelos de negocio basados en las TIC innovan con aplicaciones para generar servicios y valores agregados a los sectores de comercio y publicidad, servicios empresariales, entretenimiento, educación, cultura, seguridad y defensa.

Finalmente, con relación a la política de gobierno electrónico, al finalizar el periodo presidencial de Uribe vimos como las entidades del orden territorial solo cumplían con el $10 \%$ de la política y las entidades del orden nacional con el 55\%. El informe de gestión del MinTIC de 2014 evidencia mejoras significativas en solo cuatro años. A continuación se resaltan algunos de los indicadores presentados.

En marzo de 2014 cumplían con un nivel alto el índice de Gobierno en Línea el 52\% de las en- tidades del orden territorial y el $78 \%$ de las del orden nacional. Gracias a esto, se encuentran total o parcialmente en línea: 1.900 trámites y servicios; $57 \%$ de los trámites y servicios del orden nacional de los sectores de vivienda, educación, salud y trabajo; 569 Consejos Municipales cuentan con presencia en Internet y cumplen con los lineamientos de la estrategia; 14.478 servidores públicos y contratistas fueron sensibilizados y capacitados; 60 entidades del orden nacional y 298 alcaldías implementaron acciones de cero papel durante el 2013; se llevaron a cabo 10 ejercicios de innovación en entidades públicas para mejorar sus procedimientos internos o de servicios y $100 \%$ de los ministerios están cumpliendo con los lineamientos de redes sociales.

Teniendo en cuenta estos resultados, y aunque se han realizado grandes avances en la implementación de las rıc en Colombia, todavía existen retos por cumplir entre los que se encuentran: mejorar el nivel de cumplimiento de la política de Gobierno en Línea, aumentar el número de trámites y servicios disponibles en Internet, mejorar la reducción del consumo de papel en más entidades públicas, y desarrollar y aplicar metodologías para medir y mejorar la calidad de los servicios de Gobierno en Línea a través de canales electrónicos.

\section{CONCLUSIONES}

Como se afirmó en la introducción, la implementación de tıc en Colombia representa un esfuerzo continuo y permanente de varias administra- 
ciones, en varios periodos de gobierno, lo que muestra la existencia de políticas públicas a largo plazo, gracias a la construcción, desde un inicio, de marcos teóricos y conceptuales fuertes y coherentes que ayudan a establecer un norte para las administraciones públicas presentes y futuras.

Evidentemente el gobierno colombiano ha realizado un gran esfuerzo para promover la masificación de las TIC en todo el territorio nacional desde diferentes ángulos, esto es, desde la transformación de las instituciones públicas, la capacitación de funcionarios y ciudadanos, la construcción de infraestructura pública y la promoción del acceso a nuevas tecnologías, entre otros. Aun así, todavía es prematuro calificar el éxito o el fracaso de esta política pública, ya que los resultados solo se verán en el largo plazo conforme el país y sus ciudadanos puedan utilizar masivamente las tic en las diversas actividades de forma continua y productiva.

No obstante lo anterior, se deben resaltar cuatro factores de éxito en la implementación de la política pública de las tic. El primero es la existencia del Conpes como institución responsable de la planeación estratégica del país, porque permitió en su momento consolidar una política sobre la reforma a la administración pública y la masificación de las tıc, ayudando con esto a dar coherencia y continuidad a los programas y estrategias desarrolladas en los tres periodos presidenciales analizados.

El segundo factor de éxito es la creación de instituciones específicas como la Agenda de Co- nectividad y Consejo Nacional Digital, las cuales además de tener un alto grado de autoridad por ser integradas por funcionarios de alto rango dependientes directamente del Presidente, están bien articuladas en torno al propósito de mejorar la comunicación entre los diferentes sectores de la administración pública colombiana.

El tercer factor tiene que ver con el desarrollo de conceptos, metodologías, indicadores y sistemas de evaluación que permiten a los responsables analizar el grado de avance en la implementación de la política pública sobre gobierno electrónico, por periodos de tiempo, y determinar las mejoras y los retrocesos de forma objetiva.

Finalmente, los continuos informes de gestión de los órganos responsables de sacar adelante estas políticas ayudan a mostrar a los funcionarios públicos y a los ciudadanos el grado de avance por periodos y los principales éxitos; a consolidar la política pública por los logros alcanzados; y a visibilizar los retos que deben ser asumidos en los siguientes periodos por los equipos de trabajo, para garantizar la mejora continua de la política pública y la efectividad de las tic en la sociedad y en el Estado.

\section{Referencias}

1. Comisión Intersectorial de Políticas y de Gestión de Información de la Administración Pública [Coinfo]. (s. f.). Funciones y composición. Recuperado de: http://www.vicepresidencia.gov.co/Iniciativas/Paginas/COINFO. aspx 
2. Consejo Nacional de Política Económica y Social [Conpes]. (Julio 14 de 2011). Conpes 3701. Lineamientos de política para ciberseguridad y ciberdefensa. Recuperado de mintic: http://www.mintic.gov.co/portal/604/ articles-3510_documento.pdf

3. Consejo Nacional de Política Económica y Social [Conpes]. (Febrero 9 de 2000). Documento Conpes 3072. Agenda de Conectividad. Recuperado el 6 de abril de 2014 de mintic: http://www.mintic.gov.co/portal/604/articles-3498_documento.pdf

4. Consejo Nacional de Política Económica y Social [Conpes]. (Octubre 20 de 2003). Conpes 3248 de 2003. Renovación de la Administración Pública. Recuperado de: http://www.mintic.gov.co/portal/604/articles-3499_documento.pdf

5. Contraloría General de la República. (Agosto 5 de 2010). Informe y concepto sobre el Sistema de Control Interno de las Entidades del Estado del Orden Nacional. Vigencia Fiscal 2009. Recuperado de: contraloriagen: www. contraloriagen.gov.co/web/guest/informesconstitucionales

6. Decreto 127 de 2001 [Presidencia de la República]. Por el cual se crean las Consejerías y Programas Presidenciales en el Departamento Administrativo de la Presidencia de la República. Enero 19 de 2001. Recuperado el 19 de mayo de 2015 de mintic: http://www. mintic.gov.co/portal/604/articles-3551_documento.pdf
7. Decreto 4110 de 2004 [Presidencia de la República]. Por el cual se reglamenta la Ley 872 de 2003 y se adopta la Norma Técnica de Calidad en la Gestión Pública. Diciembre 9 de 2004. Recuperado de simege: http:// www.simege.unal.edu.co/file/Decreto $\% 20$ 4110\%202004.pdf

8. Decreto 1599 de 2005 [Presidencia de la República). Por el cual se adopta el Modelo Estándar de Control Interno para el Estado Colombiano. Mayo 26 de 2005. Recuperado de alcaldiabogota: www.alcaldiabogota.gov. co/sisjur/normas/Norma1.jsp?i=16547

9. Decreto 1151 de 2008 [Ministerio de Comunicaciones]. Por el cual se establecen los lineamientos generales de la Estrategia de Gobierno en Línea de la República de Colombia, se reglamenta parcialmente la Ley 962 de 205 y se dictan otras disposiciones. Abril 14 de 2008. Recuperado de mintic: http://www.mintic.gov.co/portal/604/articles-3643_documento.pdf

10. Decreto 2693 de 2012 [Presidencia de la República]. Por el cual se establecen los lineamientos generales de la Estrategia de Gobierno en Línea de la República de Colombia, se reglamenta parcialmente la Ley 962 de 2005, y se dictan otras disposiciones. Diciembre 21 de 2012. Recuperado de mintic: http://www.mintic.gov.co/portal/604/ articles-3586_documento.pdf.

11. Directiva Presidencial 002 de 2000. [Presidencia de la República de Colombia]. (Agos- 
to 28 de 2000). Recuperado de mintic: http://www.mintic.gov.co/portal/604/articles-3646_documento.pdf

12. Ley 790 de 2002. Por la cual se expiden disposiciones para adelantar el programa de renovación de la administración pública y se otorgan unas facultades extraordinarias al Presidente de la República. Diciembre 26 de 2002. DO n. ${ }^{\circ}$ 45046. Recuperado de mintic: http://www.mintic.gov.co/portal/604/ w3-article-3691.html

13. Ley 812 de 2003. Por la cual se aprueba el Plan Nacional de Desarrollo 2003-2006, hacia un Estado comunitario. Junio 26 de 2003. DO n. ${ }^{\circ}$ 45231. Recuperado de colciencias: http://www.colciencias.gov.co/ sites/default/files/upload/reglamentacion/ ley_812_de_2003.pdf

14. Ley 1151 de 2007. Por la cual se expide el Plan Nacional de Desarrollo 2006-2010. Julio 24 de 2007. DO n. ${ }^{\circ}$ 46700. Recuperado de alcaldiabogota: http://www.alcaldiabogota.gov.co/sisjur/normas/Norma1. jsp?i=25932

15. Ley 1450 de 2011. Por la cual se expide el Plan Nacional de Desarrollo, 2010-2014. Junio 16 de 2011. DO n. ${ }^{\circ}$ 48102. Recuperado de colciencias: http://www.colciencias.gov. co/sites/default/files/upload/reglamentacion/ley_1450_de_2011-plan_nacional_ de_desarrllo.pdf
16. Ministerio de Tecnologías de la Información y las Comunicaciones. (2011). Agenda de Conectividad. Metodología para la elaboración del diagnóstico para la implementación de la estrategia de gobierno en línea. Bogotá: Autor.

17. Ministerio de Tecnologías de la Información y las Comunicaciones. (2011a). Evolución del Gobierno en línea en Colombia. Bogotá: Autor.

18. Ministerio de Tecnologías de la Información y las Comunicaciones. (2011b). Vive Digital Colombia. Documento Vivo del Plan. Bogotá: Autor.

19. Ministerio de Tecnologías de la Información y las Comunicaciones. (2014). Informe de Gestión al Congreso de la República de Colombia 2014. Recuperado el de mintic: http://www.mintic.gov.co/portal/604/articles-6861_doc_pdf.pdf

20. Naciones Unidas. (2012). Estudio de las Naciones Unidas sobre el gobierno electrónico 2012. Gobierno electrónico para el pueblo. Recuperado de unpan: http://workspace. unpan.org/sites/Internet/Documents/ EGovSurvey2012_Spanish.pdf

21. United Nation. (2010). E-government Survey 2010. Leveraging e-government at a time of financial and economic crisis. Recuperado de unpan: https://publicadministration.un.org/egovkb/Portals/egovkb/Documents/un/2010-Survey/Cover.pdf 
22. United Nation. (2012). E-Goverment Survey 2012. New York. 2012. Recuperado de unpan: http://unpan1.un.org/intradoc/groups/public/documents/un/unpan048065.pdf

23. Universidad de los Andes. Centro de Estrategia y Competitividad. (2009). Resultados del monitoreo del Gobierno en línea en Colombia 2008. Bogotá: Ministerio de Comunicaciones/Gobierno en Línea.
24. Valencia Tello, D. C. (2015). El Estado en la era de la información y las nuevas tecnologías. Bogotá: Grupo Editorial Ibáñez.

25. World Bank. (2013). Definition of E-Government. Recuperado el 5 de julio de 2013 de worldbank: http://web.worldbank.org/ WBSITE/EXTERNAL/TOPICS/EXTINFORMATIONANDCOMMUNICATIONANDTECHNOLOGIES/EXTEGOVERNMENT/O,,contentMDK: 20507153 menuPK:702592 pagePK:148 956 piPK:216618 theSitePK:702586,00. html 